\title{
Hiperparatiroidismo primario por adenoma paratiroideo doble con glándulas paratiroides eutópica y ectópica
}

\section{Primary hyperparathyroidism due of a double parathyroid adenoma, eutopic and ectopic}

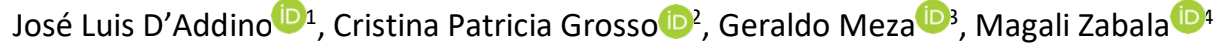 \\ 1. Hospital Privado Modelo. Hospital Municipal de Vicente López. Buenos Aires. Argentina. Correo: jldaddino@gmail.com - https://orcid.org/0000- \\ 0003-1917-6930 \\ 2. Hospital Municipal de Vicente López. Buenos Aires. Argentina. Correo: cgrosso@intramed.net https://orcid.org/0000-0003-2659-847X \\ 3. Hospital Municipal de Vicente López. Buenos Aires. Argentina. Correo: geraldomeza.sa@ hotmail.com - https://orcid.org/0000-0002-9575-5697 \\ 4. Hospital Municipal de Vicente López. Buenos Aires. Argentina. Correo: magalizabala07@gmail.com - https://orcid.org/0000-0001-7902-8169
}

Tipología: Reporte de caso clínico

Para citar este artículo: D’Addino JL, Grosso CP, Meza G, Zabala M. Hiperparatiroidismo primario por adenoma paratiroideo doble con glándulas paratiroides eutópica y ectópica. Duazary. 2021 julio; 18(3): 321-327. Doi: https://doi.org/10.21676/2389783X.4236

Recibido en mayo 31 de 2020

Aceptado en mayo 14 de 2021

Publicado en línea en agosto 03 de 2021

Palabras

clave:

hiperparatiroidi

smo primario;

paratiroidectom

ía;

enfermedades

de las

paratiroides.

\section{RESUMEN}

El hiperparatiroidismo primario (HPTP) es una afección producida por el exceso de secreción de hormona paratiroidea a partir de glándulas paratiroides que funcionan autárquicamente. Constituye la causa más común de hipercalcemia en la población. Por su embriología, las paratiroides son glándulas que pueden tener localizaciones ectópicas de forma frecuente. EI HPTP se presenta en forma esporádica en el $90 \%$ de los casos, resultante en general de un adenoma único con expansiones monoclonales de una estirpe celular única. Menos frecuentemente (2-15\%), se asocia con un segundo adenoma funcionante. La forma familiar (10\%) se asocia a síndrome de neoplasias endócrinas múltiples (NEM) como NEM 1 y NEM 2 A. Se presenta un caso de adenoma paratiroideo doble, con el segundo adenoma paratiroideo localizado en el mediastino, describiéndose los estudios preoperatorios y de localización glandular, así como los hallazgos operatorios.

Keywords:
Hyperparathyr
oidism;
primary;
Parathyroidec
tomy;
Parathyroid
Neoplasm.

Primary hyperparathyroidism (PHPT) is a condition, caused by excessive secretion of parathyroid hormone because of autonomously functioning gland. It is the most common cause of hypercalcemia in the population. Due to their embryology, parathyroid glands frequently present ectopic locations. In $90 \%$ of the cases, PHPT occurs sporadically, generally resulting from a single adenoma with monoclonal expansions of a single cell line. Less frequently (2-15\%) they are associated with a second functioning adenoma. The familial form (10\%) is associated with multiple endocrine neoplasm (MEN) syndrome, such as MEN 1 and MEN 2A. A case of double parathyroid adenoma is presented, with the second parathyroid adenoma located in the mediastinum, describing the preoperative and glandular location studies, as well as the operative findings. 


\section{INTRODUCCIÓN}

Por el desarrollo embriológico de las paratiroides, se pueden encontrar glándulas ectópicas en diferentes sectores del cuello y del mediastino ${ }^{1,2}$, lo que hace que ubicarlas constituya un desafío ${ }^{1}$. El hiperparatiroidismo primario (HPTP) suele originarse en adenomas únicos $y$, excepcionalmente, en más de una glándula. Un doble adenoma (con una glándula eutópica y una mediastinal) es muy infrecuente ${ }^{3}$, causando HPTP en $1-8 \%$ de los $\operatorname{casos}^{4-6}$.

Los pacientes suelen presentarse con astenia, litiasis renal, pancreatitis, gastritis y síndrome ulceroso, osteoporosis, entre otros. En los estudios complementarios se observan hipercalcemia, hipofosfatemia y parathormona (PTH) elevada ${ }^{2,7}$. Los métodos más específicos de localización glandular preoperatoria son el centellograma con tecnecio metoxiisobutilisonitrilo (sestamibi o MIBI) y la tomografía por emisión de positrones con flúor colina (PET/TC colina) ${ }^{1}$. La exéresis quirúrgica es el tratamiento de elección ${ }^{1,3,8}$. El objetivo de este trabajo es reportar un caso de una patología poco frecuente, con doble adenoma, uno con localización eutópica y otro con localización ectópica.

\section{REPORTE DE CASO CLÍNICO}

Paciente femenina de 53 años, remitida con diagnóstico de HPTP esporádico no asociado a neoplasia endócrina múltiple (NEM). Presentaba hipercalcemia, osteopenia, artralgias, mialgias, hipotiroidismo por tiroiditis autoinmune y litiasis renal.

Se efectuó densitometría ósea, que mostró osteopenia en raquis, antebrazos y cuello de fémures derecho e izquierdo ( $T$ Score de $-2,0,-2,0$, $-1,3$ y $-1,2$, respectivamente). La ecografía renal señalaba litiasis pielocalicial derecha $(10 \times 9 \mathrm{~mm})$. También se detectó: calcemia total de $10,6 \mathrm{mg} / \mathrm{dl}$ (VN: 8,4-10), PTH $210 \mathrm{pg} / \mathrm{ml}$ (VN: 15-65) y calcio iónico de 1,44 mmol/l (VN: 1,09-1,30); eutiroidea, con $100 \mu \mathrm{g} / \mathrm{día}$ de levotiroxina, anticuerpo antitiroperoxidasa (ATPO) $358 \mathrm{U} / \mathrm{ml}(\mathrm{VN}:<5 \mathrm{Ul} / \mathrm{ml}$ ), y función renal normal.
La ecografía cérvico-tiroidea mostró glándula tiroidea eutópica, multinodular, micronolulillar bilateral. En el tercio inferior del lóbulo derecho se evidenció imagen nodular $(12 \times 10 \mathrm{~mm})$ compatible con paratiroides derecha. El centellograma MIBI informó formación con alta probabilidad de adenoma/hiperplasia de paratiroides en sector posterior de lóbulo tiroideo derecho e imagen cerca del opérculo toráxico izquierdo (figura 1).

Se solicitaron estudios prequirúrgicos de rutina (exámenes de laboratorio, electrocardiograma y radiografía de tórax) y $\mathrm{PET} / \mathrm{TC}$ colina para mejor objetivación del opérculo torácico. En este último se observó una imagen derecha retrotiroidea, cercana al polo inferior, y otra imagen en mediastino superior izquierdo, $5 \mathrm{~cm}$ por debajo del reborde clavicular izquierdo (figura 2).

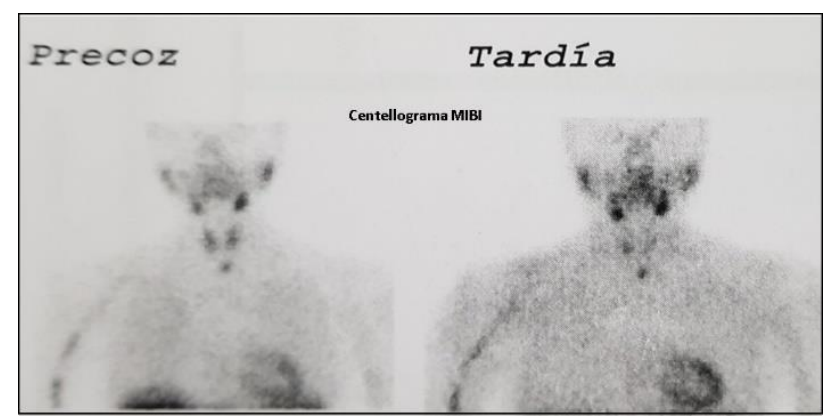

Figura 1. Centellograma MIBI, fases temprana y tardía.

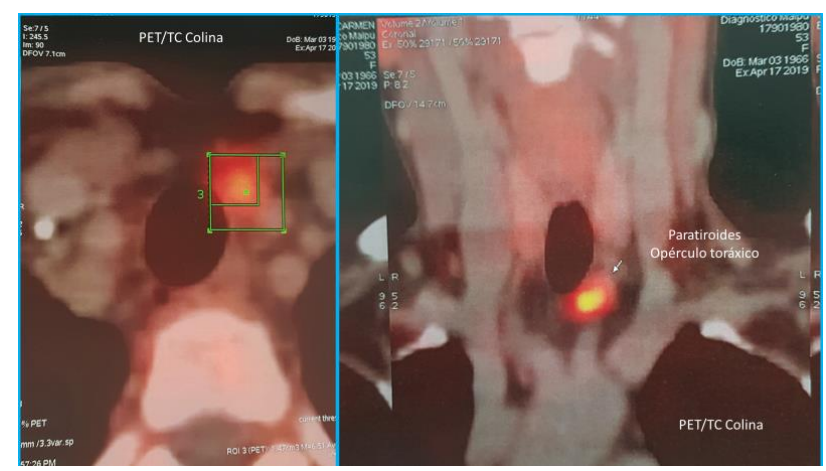

Figura 2. Tomografía por emisión de positrones con flúor colina.

Se intervino quirúrgicamente, con abordaje cervical por cervicotomía transversa, y se constató tiroides con patrón multinodular bilateral debido a tiroiditis de Hashimoto, con nódulos sólidos mayores a 0,5 $\mathrm{cm}$. Se realizó tiroidectomía, y la congelación anatomopatológica confirmó tiroiditis. A nivel retrotiroideo derecho se localizaron dos 
paratiroides: una superior, de aspecto normal, que se dejó in situ, y una inferior de $2 \mathrm{~cm}$ de diámetro que se resecó y cuya congelación confirmó adenoma paratiroideo. En la disección mediastinal, por debajo de la articulación esternoclavicular, se encontró el segundo adenoma paratiroideo, confirmado por la biopsia por congelación (figuras $3 a$ y $3 b$ ). La paciente evolucionó sin complicaciones, y fue externada a las 24 horas normocalcémica.

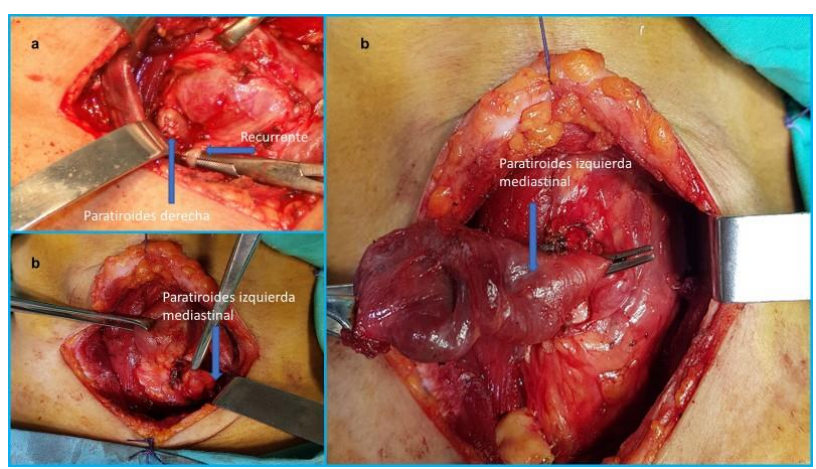

Figura 3. (a) Paratiroides derecha. (b) Paratiroides ectópica izquierda.

La anatomopatología diferida informó tiroiditis linfocitaria multinodular y dos lesiones circunscriptas, encapsuladas, conformadas por células principales y oxifílicas, sin signos de atipia: adenoma paratiroideo derecho $(1,8 \times 1,9 \mathrm{~cm})$ y adenoma paratiroideo $(2 \times 1,9 \mathrm{~cm})$ con remanente vestigial tímico en su periferia (figura 4).

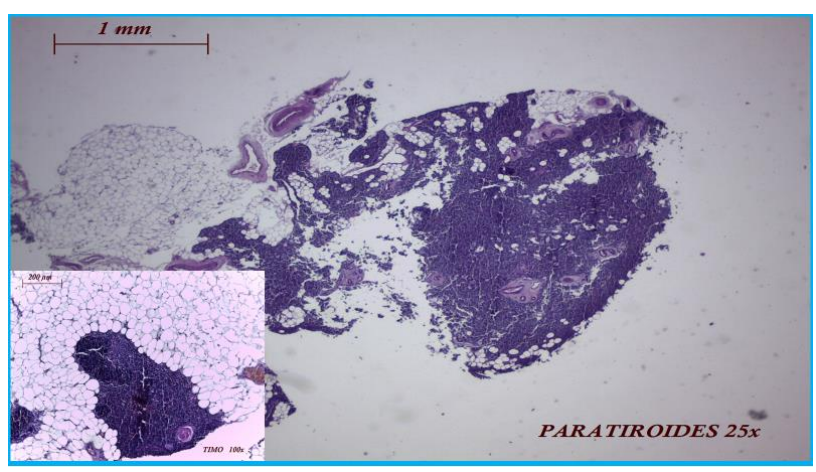

Figura 4. Paratiroides mediastinal $25 x$. Timo $100 x$ (hematoxilina-eosina).

\section{Declaración sobre aspectos éticos}

Este estudio adaptó las normas establecidas en la Declaración de Helsinki de 1975. Se presentó el consentimiento informado y se protegió el bienestar e integridad de la participante a través de la confidencialidad y el derecho a la no participación, retiro y devolución de resultados. La paciente dio su consentimiento informado para la publicación de datos de su historia clínica, así como de imágenes de sus estudios. La investigación fue avalada por el Comité de Ética del Hospital Privado Modelo de Vicente López (01-2020).

\section{DISCUSIÓN}

Históricamente se consideró al HPTP como uniglandular y al hiperparatiroidismo secundario como hiperplasia multiglandular. En 1947, Mandl $^{9}$ describió los adenomas paratiroideos dobles, con una incidencia de $2-15 \%$ sobre el total de los HPTP. No resulta fácil diferenciar una glándula aumentada de tamaño de una adenomatosa, y también es difícil para el patólogo distinguir entre adenoma e hiperplasia. Se considera a los adenomas como una neoplasia monoclonal ${ }^{10,11}$.

Habitualmente, las glándulas paratiroideas son cuatro (80-90\% de los individuos); sin embargo, el número puede ser mayor (14\%) o menor $(0,5-$ $6 \%)^{8,11}$. En el HPTP el crecimiento de las glándulas paratiroideas es autónomo, afectando más frecuentemente una glándula, o infrecuentemente dos, de forma irreversible ${ }^{12}$. Se describen dos efectos moleculares en los adenomas del HPTP ${ }^{12}$ :

1) Inversión del gen de la ciclina D1 (induce la sobreexpresión de ciclina D1, forzando la proliferación celular).

2) Mutaciones NEM-1, encontradas más usualmente en adenomas esporádicos y en los familiares.

La hiperplasia paratiroidea comienza de manera difusa, reversible si se corrige la alteración inicial; si esta persiste, se produce una mutación genética que genera nodularidad, proliferación celular y autonomía glandular ${ }^{13}$. Macroscópicamente, las glándulas se observan aumentadas de tamaño. Microscópicamente, los adenomas suelen ser capsulados con predominio de proliferación de células principales, distribución difusa o nodular, aisladas células oxifílicas y escasos adipocitos ${ }^{12}$. 
Clínicamente, resulta casi imposible diferenciar un adenoma único de un adenoma doble. Algunos autores postulan que el adenoma doble es más frecuente en mujeres en la quinta/sexta década de la vida, y se asociaría con mayor sintomatología ósea y muscular, y PTH más elevada que la hallada en los adenomas únicos ${ }^{14}$. La hipercalcemia en los adenomas dobles sería más estable, con incremento lento, a diferencia de las hiperplasias o los carcinomas paratiroideos ${ }^{15}$.

Debido a la embriología de las paratiroides, la principal dificultad que presenta la cirugía radica en la diversidad de su ubicación. Las glándulas superiores, originadas de la cuarta bolsa faríngea, migran adheridas a la tiroides; por esta razón es más frecuente encontrarlas en su cara posterosuperior. El timo y las paratiroides inferiores se originan de la tercera bolsa faríngea. Por este motivo, pueden localizarse desde la bifurcación carotídea hasta la parte lateral e inferior de la tiroides. Este recorrido embriológico determina mayor posibilidad de localizaciones ectópicas ${ }^{1,16}$.

Las glándulas paratiroideas superiores se localizan en la mitad superior del tercio medio de la glándula tiroides ( $83 \%$ de los casos) y en el tercio superior ( $9 \%$ de los casos). Algunos autores las citan en la unión cricotiroidea (77\% de los casos) y en el tercio superior, sobre la cara posterior de la glándula tiroides (22\% de los casos) y siempre más dorsalmente que las inferiores ${ }^{17}$. Las glándulas paratiroideas superiores ectópicas se ubican por debajo de la bifurcación carotidea, en dirección inferoposterior, hacia el mediastino posterosuperior, en el espacio retrofaríngeo y traqueoesofágico ${ }^{17}$. Las paratiroides inferiores se encuentran cercanas a la región posterolateral de la tiroides, en el origen de la arteria tiroidea inferior ( $77 \%$ de los casos) y cercanas al polo inferior tiroideo (13\% de los casos). El resto se hallan en el timo o a lo largo del recorrido embriológico, desde el ángulo mandibular hasta el pericardio ${ }^{17}$ (figura 5).

Debido al tamaño de las paratiroides y su ubicación y número variables, la localización suele ser dificultosa en manos no experimentadas. La presencia concomitante de patología tiroidea dificulta la diferenciación entre un nódulo tiroideo y una glándula paratiroidea patológica, lo que da lugar a falsos positivos ${ }^{19,20}$.

Entre los estudios de localización utilizados, sin mencionar la ecografía (actualmente rutinaria y operador dependiente), se cita el centellograma MIBI. El tejido paratiroideo tiene mucha densidad mitocondrial, y el tecnecio metoxiisobutilisonitrilo (sestamibi o MIBI) se acumula en las mitocondrias por ser lipofílico y especialmente en las células oxifílicas de los adenomas paratiroideos ${ }^{21}$. Esto resulta en una limitante del estudio ya que otros tejidos captantes, como adenomas tiroideos, pueden resultar en falsos negativos ${ }^{21,22}$. La sensibilidad del método es del $60-90 \%$, y la especificidad es del $30 \%{ }^{21}$.

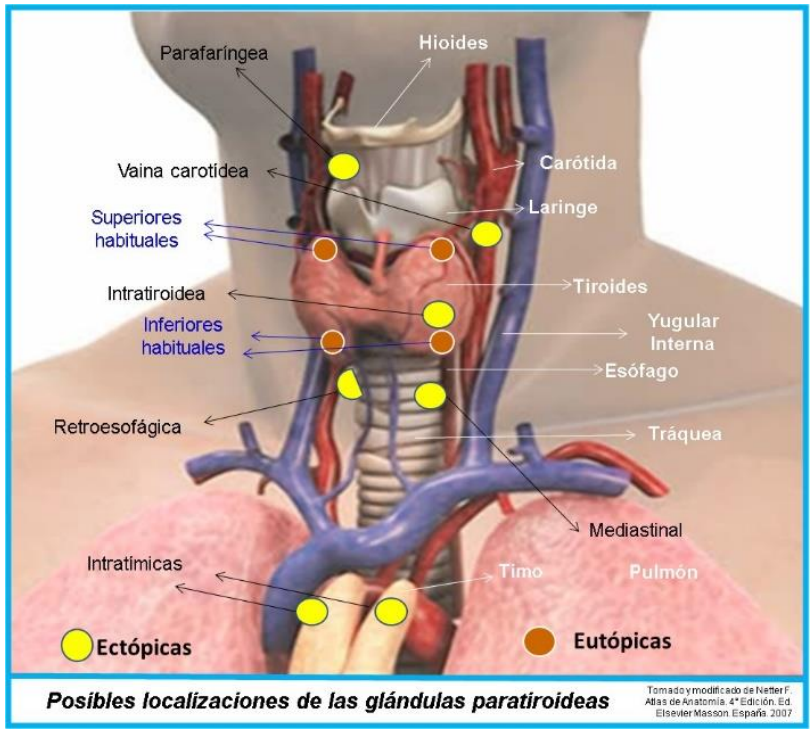

Figura 5. Localización eutópica y ectópica de las glándulas paratiroides. Fuente: tomado y modificado de Netter et $a l^{18}$.

La PET es un método de diagnóstico no invasivo basado en imágenes tomográficas que se obtienen del rastreo de isótopos emisores de positrones de vida media ultracorta administrados al paciente. El equipo mide la actividad metabólica y detecta la distribución tridimensional del radiofármaco usado. Los detectores generan una imagen al captar los fotones que emite el paciente, los cuales resultan de la aniquilación del positrón emitido por el radiofármaco y el electrón que produce el cuerpo humano. 
La fosfatidilcolina interviene en la síntesis de la membrana celular, y ciertos tejidos, especialmente los tumorales, presentan una alta tasa metabólica en la membrana celular. Este radiofármaco fue reportado como útil para las glándulas paratiroideas luego de que incidentalmente se descubriera una hiperplasia paratiroidea en un paciente con un cáncer de próstata ${ }^{23}$, y se ha reportado que ingresa a las células por difusión facilitada a través de los canales transportadores ${ }^{24}$. El incremento de la proliferación celular y del metabolismo en las hiperplasias aumentaría la absorción de colina, la cual, por la fosforilación de la colina kinasa, queda atrapada en los fosfolípidos de la membrana, formando la fosfatidilcolina. Basado en el mecanismo de acción de la colina, el 18Ffluorocolina resultaría entonces un análogo de la colina, útil para la evaluación de las paratiroides ${ }^{25}$. La 18F-fluorocolina emite un positrón con una vida media de 109,7 min y una energía de $1.656 \mathrm{MeV}$. Además, se metaboliza y elimina por excreción urinaria $^{25}$. La sensibilidad es del $92 \%$, y la especificidad para detectar glándulas paratiroideas anómalas es del $80 \%{ }^{26,27}$.

Existen diferentes técnicas para identificar la presencia de glándulas paratiroideas en el intraoperatorio. Se han utilizado luz cercana al infrarrojo, colorantes fluorescentes, azul de metileno, ácido aminolevulínico o verde de indocianina y la sonda gamma cámara ${ }^{28}$. No obstante, ninguno es más sensible que la experiencia del cirujano ${ }^{1}$. La determinación de PTH intraoperatoria (PTHio) es útil para predecir el éxito de la paratiroidectomía en el HPTP si los niveles de PTHio descienden un $50 \%$ respecto al basal, pero no es útil para la localización glandular ${ }^{29,30}$.

Los estudios de localización preoperatoria son importantes para facilitar la ubicación y disminuir el tiempo operatorio. Sin embargo, ninguno de los estudios disponibles en la actualidad (ecografía, tomografía computada, resonancia magnética nuclear, centellograma MIBI y PET/TC colina) poseen la sensibilidad suficiente para ser utilizados como único método diagnóstico, por lo que se aconseja combinarlos con el juicio de un cirujano experimentado ${ }^{1,29,30}$.

\section{CONCLUSIÓN}

El HPTP es generalmente ocasionado por un adenoma único, pero no es infrecuente el hallazgo de adenomas múltiples. En éstos últimos, la clínica es inespecífica, y los métodos más específicos de localización glandular preoperatoria - centellograma MIBI y PET/TC colina- no tienen mayor sensibilidad que la exploración cervical por un cirujano experimentado.

\section{DECLARACIÓN SOBRE CONFLICTOS DE INTERESES}

Los autores declaran que no existe conflicto de intereses

\section{CONTRIBUCIÓN DE LOS AUTORES}

Primer autor: trabajo de campo, cirujano interviniente, diseño metodológico.

Segundo autor: trabajo de campo, seguimiento endocrinológico y análisis bibliográfico.

Tercer autor: redacción y búsqueda bibliográfica. Cuarto autor: redacción.

\section{REFERENCIAS BIBLIOGRÁFICAS}

1. D'Addino J, Grosso C. Hiperparatiroidismo Secundario. Controversias [Internet]. Primera edición. Berlín: Editorial Académica Española; 2019. Disponible en: https://www.amazon.com//es/Jos\%C3\%A9-Luis-DAddino/dp/6200017700

2. Avello Canisto F. Hiperplasia paratiroidea ectópica de doble localización, intratiroidea e intratorácica. An Fac Med. 2013; 74(4): 335-8. Doi: https://doi.org/10.15381/anales.v74i4.2710

3. Cáceres D, Restrepo C. Doble adenoma de paratiroides como presentación de hiperparatiroidismo primario y detalles de su tratamiento. Acta Med Colomb. 2016; 41(3): 198201. Doi: https://doi.org/10.36104/amc.2016.720

4. Baloch Z, LiVolsi. Double adenoma of the parathyroid gland does the entity exist? Arch Pathol Lab Med. 2001; 125(2): 178-9. Doi: 10.5858/2001125-0178-DAOTPG 
5. Bergson E, Heller K. The clinical significance and anatomic distribution of parathyroid double adenomas. J Am Coll Surg. 2004; 198(2): 185-9. Doi: https://doi.org/10.1016/j.jamcollsurg.2003.09.012

6. De Gregorio L, Lubitz C, Hodin R, Gaz R, Parangi S, Phitayakorn $\mathrm{R}$, et al. The Truth about Double Adenomas: Incidence, Localization, and Intraoperative Parathyroid Hormone. J Am Coll Surg. 2016; 222(6): 1044-52. Doi: https://doi.org/10.1016/j.jamcollsurg.2015.12.048

7. Goñi I, Campusano C, Gejman R, Orellana P. Hiperparatiroidismo primario de origen multiglandular con glándula paratiroides intratiroidea hiperfuncionante. Rev Chil Cir. 2011; 63(4): 418-21. Doi: https://doi.org/10.4067/S071840262011000400015

8. Olivera Fajardo D, Puerto Lorenzo J, Torres Ajá L. Adenoma paratiroideo. Presentación de un caso. MediSur [revista en la Internet]. 2016; 14(5): 567$72 . \quad$ Disponible en: http://scielo.sld.cu/scielo.php?script=sci_arttext\&p $\mathrm{id}=\mathrm{S} 1727-897 \mathrm{X} 2016000500013$

9. Mandl F. Hyperparathyroidism; a review of historical developments and the present state of knowledge on the subject. Surgery [revista en la Internet]. 1947; 21(3): 394-440. Disponible en: https://pubmed.ncbi.nlm.nih.gov/20290625/

10. Ghandur-Mnaymneh L, Kimura N. The parathyroid adenoma. A histopathologic definition with a study of 172 cases of primary hyperparathyroidism. Am J Pathol. 1984; 115(1): 70$83 . \quad$ Disponible en: https://www.ncbi.nlm.nih.gov/pmc/articles/PMC19 00356/

11. Loyd R, Osamura Roert Y, Klopper G, Rosai J. WHO Classification of Tumors of Endocrine Organs [Internet]. Cuarta edición. Lyon, Francia: International Agency for Research on Cancer; 2017. Disponible en: https://publications.iarc.fr/BookAnd-Report-Series/Who-Classification-OfTumours/WHO-Classification-Of-Tumours-OfEndocrine-Organs-2017
12. Maitra A. Sistema Endocrino. En: Elsevier Inc. Robbins y Cotran. Patología estructural y funcional [Internet]. Novena edición. Barcelona, España: Editorial Elsevier España; 2015. p. 1100-5. Disponible en: https://dspace.scz.ucb.edu.bo/dspace/handle/123 456789/21365

13. Hómez B. Actualización en el tratamiento del hiperparatiroidismo. Revista Venez Endocrinol Metab [revista en la Internet]. 2008; 6(3): 6-12. Disponible en: http://www.scielo.org.ve/scielo.php?pid=S169031102008000300003\&script=sci_arttext

14. Tezelman S, Shen W, Shaver J, Siperstein A, Duh $\mathrm{Q}$, Klein $\mathrm{H}$, et al. Double Parathyroid Adenomas. Clinical and Biochemical Characteristics Before and After Parathyroidectomy. Ann Surg. 1993; 218(3): 300-9. Doi: https://doi.org/10.1097/00000658199309000-00009

15. Peña Irún A, González Santamaría A, Fontanillas Garmilla N, Arenal Barquín S. El adenoma paratiroideo también puede ser una urgencia. Semergen. 2011; 37(2): 99-101. Doi: https://doi.org/10.1016/j.semerg.2010.11.007

16. Langman J. Embriología médica [Internet]. Décimo cuarta edición. México: Editorial Interamericana; 2019. Disponible en: https://www.edicionesjournal.com/Papel/9788417 602116/Langman+Embriolog\%C3\%ADa+M\%C3\%A9 dica $+E d+14 \%$ C $2 \% B A$

17. Perinetti H. Hiperparatiroidismo primario, secundario y terciario: actualización. Instituto de Patología de la Tiroides. Departamento de Medicina Quirúrgica. F.C.M. UN Cuyo. Rev Médica Univer [revista en la Internet]. 2005 [citado 2020 Marzo]; 1(1): $\quad 10-28$ Disponible en https://videlarivero.bdigital.uncu.edu.ar/objetos_digitales/1238/ perinettirmuv1n1.pdf

18. Netter F. Cabeza y Cuello. Glándula Tiroides y Laringe. En: Elsevier Masson. Atlas de Anatomía Humana [Internet]. Cuarta Edición. Barcelona, España: Editorial Elsevier Masson; 2007. p. 94-96. 
Disponible en: https://www.casadellibro.com/libroatlas-de-anatomia-humana-4-

ed/9788445817599/1143815

19. Ferrer Ramírez M, Amorós Sebastiá L, Cano Terol C, Caballero Calabuig E, Hernández Mijares A, López Martínez R. Valor diagnóstico de las técnicas de localización paratiroidea en la cirugía del hiperparatiroidismo primario. Acta Otorrinolaringol Esp. 2003; 54(3): 220-4. Doi: https://doi.org/10.1016/S0001-6519(03)78407-6

20. Arkles L, Jones T, Hicks R, De Luise M, Chou S. Impact of complementary parathyroid scintigraphy and ultrasonography on the surgical management of hyperparathyroidism. Surgery. 1996; 120(5): 84551. Doi: https://doi.org/10.1016/S00396060(96)80093-6

21. Berber E, Parikh N, Ballen N, Garner C, Siperstein A. Factors Contributing to Negative Parathyroid Localization: An Analysis of 1000 patients. Surgery. 2008; 144(1): 74-9. Doi: https://doi.org/10.1016/j.surg.2008.03.019

22. Markarian M, Yelin G, Aparicio R, Marino J. Utilidad del centellograma TC 99-Sestamibi en la detección de adenomas e hiperplasias de glándulas paratiroides. Rev Argent Radiol [revista en la Internet]. 2005 [citado 2020 Marzo]; 69(2): 77-81. Disponible en: https://www.redalyc.org/pdf/3825/382538435003. pdf

23. Cazaentre T, Clivaz F, Triponez F. False-Positive Result in 18F-Fluorocholine PET/CT Due to Incidental and Ectopic Parathyroid Hyperplasia. Clin Nucl Med. 2014; 39(6): e328-30. Doi: https://doi.org/10.1097/RLU.0b013e3182a77b62

24. Cardona Arboniés J, Rodríguez Alfonso B, Mucientes Rasilla J, Martínez Ballesteros C, Zapata Paz I, Prieto Soriano A, et al. La PET/TC con F-Colina en la estadificación y recidiva bioquímica de pacientes con cáncer de próstata: cambios en la clasificación y planificación de radioterapia. Rev Esp Med Nucl Imagen Mol. 2017; 36(5): 292-7. Doi: https://doi.org/10.1016/j.remn.2017.02.007
25. Prabhu M, Damle N. Fluorocholine PET imaging of parathyroid disease. Indian J Endocr Metab. 2018; 22(4): 535-41. Doi: https://doi.org/10.4103/ijem.IJEM_707_17

26. Lezaic L, Rep S, Sever M, Kocjan T, Hocevar M, Fettich J. ${ }^{18}$ F-Fluorocholine PET/CT for localization of hyperfunctioning parathyroid tissue in primary hyperparathyroidism: a pilot study. Eur J Nucl Med Mol Imaging. 2014; 41: 203-9. Doi: https://doi.org/10.1007/s00259-014-2837-0

27. Michaud L, Balogova S, Burgess A, Ohnona J, Huchet V, Kerrou K, et al. A Pilot Comparison of 18Ffluorocholine $\mathrm{PET} / \mathrm{CT}$, Ultrasonography and 123I/99mTc-sestaMIBI Dual-Phase Dual-Isotope Scintigraphy in the Preoperative Localization of Hyperfunctioning Parathyroid Glands in Primary or Secondary Hyperparathyroidism: Influence of Thyroid Anomalies. Medicine (Baltimore). 2015; 94: e1071

Doi: https://doi.org/10.1097/MD.0000000000001701

28. Falco J, Dip F, Quadri P, de la Fuente M, Prunello $M$, Rosenthal R. Increased identification of parathyroid glands using near infrared light during thyroid and parathyroid surgery. Surg Endosc [revista en la Internet]. 2017; 31: 3737-42. Doi: https://doi.org/10.1007/s00464-017-5424-1

29. Cabo J, Gallagher K, Baregamian N. Challenges and controversies in the surgical management of uremic hyperparathyroidism: A systematic review. Am J Surg. 2018; 216(4): 713-22. Doi: https://doi.org/10.1016/j.amjsurg.2018.07.030

30. D’Addino J, Caubet M, Pigni M, Mayorga H. La localización del $10 \%$ de las glándulas patológicas en el hiperparatiroidismo secundario dependen de la experiencia del cirujano. Rev. Duazary. 2015; 12(2): 125-32.

Doi: 